phorylation is centrally involved in host signal transduction and communication with the cytoskeleton. Thus it should come as no surprise that microbial pathogens have evolved mechanisms to affect this pathway. In the near future this avenue of research should improve our understanding of both microbial pathogenesis and eukaryotic pathways of signal transduction.

Daniel A. Portnoy and Gregory A. Smith are in the Department of Microbiology, University of Pennsylvania School of Medicine, Philadelphia, Pennsylvania 191046076, USA.

\title{
QUANTUM OPTICS
}

\section{New spectroscopic frontiers}

\section{Stephen M. Barnett}

SPECTROSCOPISTS may be able to squeeze improved sensitivity out of their spectrometers by using a novel state of light. E. S. Polzik, J. Carri and H. J. Kimble (Phys. Rev. Lett. 68, 3020-3023; 1992) show how it is possible to reduce noise levels below the quantum limit using a new, tunable source of so-called squeezed light, surpassing even the best laser systems.

The introduction of lasers and the ensuing development of nonlinear optical techniques have revolutionized spectroscopy. This revolution has been due to the unique properties of laser light; it is coherent, intense and, of particular relevance to spectroscopy, it can be made highly monochromatic, stable and tunable. Moreover, laser light is very 'clean', in that it does not have the large intensity and phase fluctuations associated with conventional light. Yet even lasers do not represent the ultimate in clean light sources, as Polzik et al. show.

The question facing spectroscopists is how accurately they can detect the absorption of light. The answer depends upon both the degree of precision with which they can determine the level of light impinging on the sample and the efficiency with which they can detect the unabsorbed light. Polzik et al. tackle the problem in two steps. Frequencymodulated spectroscopy, a subtle trick of the trade to make the most of laser light, addresses the first step by concentrating on the differences in the absorption and phase shifts experienced by light at different frequencies, which are easier to handle than absolute levels at a single frequency.

The modulation of the laser light produces sidebands at frequencies either side of the original laser frequency. The differential absorption experienced by these sidebands converts the frequency modulation to amplitude modulation. This is detectable in the beat note between the sidebands and the carrier at the original laser frequency.

But the sensitivity of this technique is limited by the precision with which one can ensure that the two sidebands have the same intensity. Laser light can be made highly coherent and stable, but even after all sources of technical noise have been removed there remains an intrinsic quantum noise. This residual noise is responsible for poissonian fluctuations in the detector photocurrent. Using this photocurrent to infer the intensity, one finds an uncertainty that is proportional to the square root of the mean intensity. The precision with which one can specify intensities or intensity differences is ultimately limited by this uncertainty - the standard quantum limit. Frequency-modulated spectroscopy with lasers can attain but not exceed this limiting sensitivity, which is where squeezed light comes in.

The origin of the standard quantum limit lies in the quantum nature of the electromagnetic field. In quantum optics the sinusoidal and cosinusoidal oscillating components of the field are incompatible. This is analogous to the incompatibility of the position and momentum of a particle in quantum mechanics. Heisenberg's uncertainty principle places bounds on the precision with which we can discern both the position and momentum of the particle. It is possible to improve one's knowledge of the particle's position but only at the expense of losing information about its momentum. In the same way spectroscopists are restricted in the extent to which they can determine both components or quadratures of the electromagnetic field. All field states are similarly restricted, even the vacuum. The uncertainty in the field quadratures is the origin of the quantum fluctuations responsible for the standard quantum limit.

Laser light has an equal level of uncertainty in both quadratures of the field. However, squeezed light has one quadrature more precisely specified at the expense of increased uncertainty in the other. The level of uncertainty in the reduced-noise field component or squeezed quadrature is even less than that associated with the vacuum. When squeezed light is superposed on laser light, the resulting beam has reduced intensity or phase fluctuations, depending upon the phase of the original laser beam. If the laser light is in phase with the squeezed quadrature then the resulting beam has reduced intensity fluctuations. But if they are out of phase, the level of intensity noise is increased and the phase fluctuations are reduced.

Polzik, Carri and Kimble exploit this suppression of quantum noise as the second element in their experiment. By generating squeezed light with reduced intensity fluctuations they have shown that it is possible to increase the signalto-noise ratio beyond the standard quantum limit. The lower level of intensity uncertainty means that small changes of intensity due to weak absorption can be measured more accurately. Moreover, by reducing the background quantum noise level, weaker spectroscopic features may become discernible.

Central to spectroscopic applications is the requirement for tunable light. Polzik et al. have developed the first tunable source of squeezed light for their experiment. Its heart is a powerful titaniumsapphire laser (itself a recent development) which produces blue light. This light is passed through a crystal of potassium niobate in a resonant optical cavity, where some of its photons are converted into photon 'twins' of half the frequency. These make the tunable squeezed light at a wavelength of around $855 \mathrm{~nm}$, which has photocurrent noise fluctuations 5 decibels (about a factor of three) below the standard quantum limit. This level of squeezing is retained as the source is tuned over 2 gigahertz. The squeezed light is mixed with frequency-modulated laser light to give a probe beam with reduced quantum noise. Squeezing the quantum noise associated with the sidebands reduces the level of noise seen in the beat note (and so improves the signal-to-noise ratio) by more than a factor of two.

Enhanced spectroscopic sensitivity is not the only benefit offered by squeezing light. Reducing the level of phase fluctuations instead of the intensity noise can improve the sensitivity of interferometric measurements. The ability to reduce the noise level of modulated light, central to the new development in frequency-modulated spectroscopy, has potential in optical communications. Squeezed light can modify fundamental radiative processes such as spontaneous emission. It might be possible to induce ultranarrow spectroscopic features by detecting the decay of atoms with squeezing-reduced natural linewidths. Tunable squeezed light is the key to unlocking a new frontier in spectroscopic precision.

Stephen M. Barnett is in the Department of Physics and Applied Physics, John Anderson Building, University of Strathclyde, Glasgow G4 ONG, UK. 